\title{
Reflectance anisotropy spectroscopy: A probe to explore organic epitaxial growth
}

\author{
G. Bussetti, ${ }^{\text {a) }}$ S. Cirilli, A. Violante, V. Chiostri, C. Goletti, and P. Chiaradia \\ Dipartimento di Fisica and CNISM, Università degli Studi di Roma "Tor Vergata," I-00133 Roma, Italy
}

\begin{abstract}
A. Sassella, M. Campione, b) L. Raimondo, D. Braga, and A. Borghesi
Dipartimento di Scienza dei Materiali and CNISM, Università degli Studi di Milano-Bicocca, I-20125 Milano, Italy
\end{abstract}

(Received 1 December 2008; accepted 26 May 2009; published 30 June 2009)

\begin{abstract}
Reflectance anisotropy spectroscopy (RAS) is demonstrated to be particularly suitable for studying the deposition of organic epitaxial layers in ultrahigh vacuum by organic molecular beam epitaxy, thanks to its high sensitivity and applicability in situ. In the case of $\alpha$-quaterthiophene, both homoepitaxy and heteroepitaxy have been monitored, demonstrating the crystallinity of the films up to tens of monolayers and the epitaxial relation to the substrate. In both cases, optical RAS data are compared to the results of ex situ characterization of the same samples by atomic force microscopy. (C) 2009 American Vacuum Society. [DOI: 10.1116/1.3155399]
\end{abstract}

\section{INTRODUCTION}

In recent years the research in solid state physics has been typified by a remarkable interest in molecular organic materials, whose properties can be tailored to the rational design of highly functional organic electronic devices. The combination of different organic semiconductors in multilayer structures has been demonstrated to offer several benefits in order to improve device function and efficiency, as in the case of organic field effect transistor (OFET), light emitting OFETs, ambipolar OFETs, and solar cells. ${ }^{1}$ The architecture of these devices necessarily requires the use of thin films, and therefore the presence of interfaces-being the natural boundaries between different materials: organic/organic and organic/inorganic - is often a key point determining the device performance. The detailed investigation of the interface development process is thus at the focus of research, reproducing a situation already experienced in inorganic surface science about 20 years ago. Undeniably, the surface science community has brought new insights into the study of organic materials, e.g., defining how molecules assemble on a solid surface to form a thin film and characterizing the morphology and electronic properties of interfaces. In this respect, the possibility of growing crystalline organic layers in epitaxial conditions and the concept itself of epitaxy for organic materials have been deeply debated., ${ }^{2,3}$

In organic molecular beam epitaxy (OMBE) - the most efficient and reliable method to develop prototypical organic systems - the interface formation can be achieved step by step from the very early stages (submonolayer coverage) up to the complete growth. ${ }^{2,3}$ This means that in OMBE we have the opportunity to monitor in real time the interface formation by applying an appropriate spectroscopy, provided it does not interfere with growth. It has been largely demon-

\footnotetext{
a) Author to whom correspondence should be addressed; electronic mail: bussetti@roma2.infn.it

${ }^{b)}$ Present address; Department of Geological Sciences and Geotechnologies, Università degli Studi di Milano Bicocca, I-20126 Milano, Italy.
}

strated that optical spectroscopies in particular, reflectance anisotropy spectroscopy (RAS) ${ }^{4}$ and surface differential reflectance ${ }^{5}$-possess the proper features to this aim, ${ }^{6-9}$ avoiding contaminants and damage to the sample, being applicable in vacuum and on insulating samples, giving the possibility to study buried interfaces and various surface morphologies, and finally (in the case of RAS) being sensitive to the anisotropic optical properties of organic materials. In this article, we substantiate the capability of RAS to study the evolution of two different organic-organic interfaces during OMBE growth: $\alpha$-quaterthiophene $(\alpha-4 \mathrm{~T})$ onto a single crystal of the same material (example of homoepitaxy) and $\alpha$-quaterthiophene onto a single crystal of potassium hydrogen phthalate (KAP) (example of heteroepitaxy). In both cases, the optical data demonstrate the epitaxial growth and show how the crystalline properties of the layer are preserved up to higher thickness.

\section{EXPERIMENT}

$\alpha$-4T was synthesized and purified according to the procedure reported in Ref. 10. Single crystals of the lowtemperature polymorph of $\alpha-4 \mathrm{~T}(\alpha-4 \mathrm{~T} / \mathrm{LT})$ have been grown from solution by the floating-drop method, ${ }^{11}$ obtaining crystals with size up to a few $\mathrm{cm}^{2}$ and controlled thickness. A few micron thick crystals, as measured by a Digital Instrument Dektak 8 profilometer, have been selected for the optical and morphological characterization and placed on fused silica or on a high value refractive index glass substrate. $\alpha-4 \mathrm{~T} / \mathrm{LT}$ crystal structure belongs to the monoclinic system with unit cell parameters of $a=6.085 \AA, b=7.858 \AA$, $c=30.483 \AA, \beta=91.81^{\circ},{ }^{12}$ and 4 molecules/ unit cell. The molecules are arranged in the crystal following a herringbone motif, forming layers parallel to the (001) plane (the herringbone plane, which is parallel to the exposed surface), thus exhibiting stronger intermolecular interactions within this plane compared to those between other planes. 
The $\alpha-4 \mathrm{~T}$ thin films were grown on the (001) face of $\alpha$-4T/LT single crystals and on (010)-oriented KAP in ultrahigh vacuum (UHV) by OMBE at a base pressure below 5 $\times 10^{-10}$ Torr, with $165^{\circ} \mathrm{C}$ deposition temperature and $0.2 \mathrm{~nm} / \mathrm{min}$ deposition rate. The source was a Knudsen-type effusion cell with a double heater and a double temperature control; a quartz microbalance calibrated for $\alpha-4 \mathrm{~T}$ and installed close to the substrate was used to dose the material and to measure the nominal film thickness. ${ }^{13}$ The substrates have been maintained at room temperature $(\alpha-4 \mathrm{~T} / \mathrm{LT}$ single crystals) or at $146 \mathrm{~K}$ (KAP) during the deposition process. The structure of the $\alpha-4 \mathrm{~T}$ films grown at room temperature has been identified ${ }^{14,15}$ as that of $\alpha$-4T/LT single crystals.

The RAS experiments reported here were carried out in situ at near-normal incidence with a homemade apparatus with two polarizers (one polarizer plus one analyzer) in the spectral range of $1.5-5.0 \mathrm{eV} .^{6}$ The RAS signal is expressed, as a function of the photon energy, in terms of

$$
\frac{\Delta R}{R}=2 \frac{R_{\alpha}-R_{\beta}}{R_{\alpha}+R_{\beta}},
$$

where $R_{\alpha}$ and $R_{\beta}$ are the light intensities reflected by the sample for incident light linearly polarized along two orthogonal directions $\alpha$ and $\beta$. The substrates have been mounted by aligning $\alpha(\beta)$ nearly parallel to the $b_{\alpha-4 \mathrm{~T}}\left(a_{\alpha-4 \mathrm{~T}}\right)$ axis of the $\alpha$-4T/LT crystal substrate or to the $a_{\mathrm{KAP}}\left(c_{\mathrm{KAP}}\right)$ axis of the KAP crystal substrate. The axis directions on the crystal are determined by polarized optical microscopy with an uncertainty of $\pm 2^{\circ}$; the alignment of the sample mounted on the OMBE chamber with respect to directions $\alpha$ and $\beta$ is slightly worst.

Near-normal incidence reflectance spectra were collected in the spectral range from 2 to $5 \mathrm{eV}$ on the (001) surface of $\alpha$-4T single crystals using a spectrometer PerkinElmer Lambda900 with polarized light (depolarizer and GlanTaylor calcite polarizers); all spectra were corrected for the instrumental spectral response.

Atomic force microscopy (AFM) measurements were carried out ex situ using a Digital Instruments Nanoscope Illa. Intermitting contact mode with silicon cantilevers (resonance frequency of $300 \mathrm{kHz}$ and force constant of $40 \mathrm{~N} / \mathrm{m}$ ) was employed for collecting images on a micrometer scale, whereas atomic-scale images were collected in contact mode with single beam silicon nitride cantilevers (force constant of $0.05 \mathrm{~N} / \mathrm{m})$.

\section{APPLICATION OF RAS TO ORGANIC EPITAXY}

Molecular crystals are, in general, highly anisotropic and exhibit optical properties strongly dependent on the propagation direction and the polarization of the incident light. This suggests that optical spectroscopy under polarized light represents the natural choice for the investigation of their optical properties; nevertheless, the optical anisotropy of the organic crystals used as substrate in OMBE growth complicates the interpretation of RAS data, so that well-assessed results for inorganic isotropic crystals cannot be usually borrowed to organics without additional analysis. The well known three- layer model ${ }^{16}$ usually applied to obtain from RAS data the dielectric function of a thin layer deposited onto an isotropic substrate does not hold, in general, for organic layers deposited onto anisotropic organic crystals. ${ }^{17}$ Hence, a detailed knowledge on the anisotropic optical properties of the pristine substrate is fundamental for a correct interpretation of the RAS spectra. Since, at the moment, quantitative results based on a simple and direct model (as the abovementioned three-layer model) cannot routinely be achieved, a phenomenological approach is necessary: in this way, fundamental information on the epitaxial growth of organic materials has been obtained, as the assessment of the organic overlayer crystallinity or the epitaxial relationship between the overlayer and the substrate.

Other experimental techniques, such as AFM or scanning tunnelling microscopy, could actually give more directly similar or even more detailed results, but RAS offers special benefits when applied as in situ technique during the deposition of organic layers (for example, during the OMBE growth of organic-organic heterojunctions). This growth monitoring gives the chance to probe the real-time formation of a crystalline layer and of the interface with the substrate, the evolution of its optical response with increasing thickness, and the role of deposition conditions (temperature, pressure, and environment), then to detect minute changes in the molecular orientation, relaxation at the interface, or postgrowth phenomena. ${ }^{17,18}$ As RAS is a modulation spectroscopy, it possesses an extremely high sensitivity and time stability, offering the opportunity to detect even the tiny variation in the anisotropy signal generated by the OMBE deposition of a fraction of a monolayer. ${ }^{6}$ Finally, as the optical response of the growing samples is monitored over $\mathrm{mm}^{2}$-sized regions, information on its average behavior is obtained, which is often more interesting for device development than local properties.

To elucidate the power and to substantiate the utility of RAS when applied to organic epitaxy, in this section we will report results about the growth of $\alpha$-4T films on different organic substrates: in particular, we will present the homoepitaxial and heteroepitaxial growth of $\alpha-4 \mathrm{~T} / \mathrm{LT}$, respectively, onto a $\alpha$-4T/LT single crystal and onto a (010)oriented KAP crystal. We will divide the discussion in three parts: (i) analysis of the RAS spectrum of $\alpha$-4T/LT single crystals (used as substrate in Sec. III B), (ii) RAS study of organic homoepitaxy, and (iii) RAS study of organic heteroepitaxy ( $\alpha$-4T onto KAP).

\section{A. Single crystals}

The optical properties of $\alpha$-4T/LT crystals are well described on the basis of the Frenkel-Davydov exciton theory, ${ }^{19}$ which predicts four crystal bands originating from the first molecular transition, only two of them being optically accessible due to selection rules: the transition to the lower energy band exhibits a dipole moment parallel to the $b$ axis, while the direction of the dipole moment of the transition to the higher energy level lies in the (010) plane, being tilted by about $27^{\circ}$ with respect to the normal (001) plane. As 

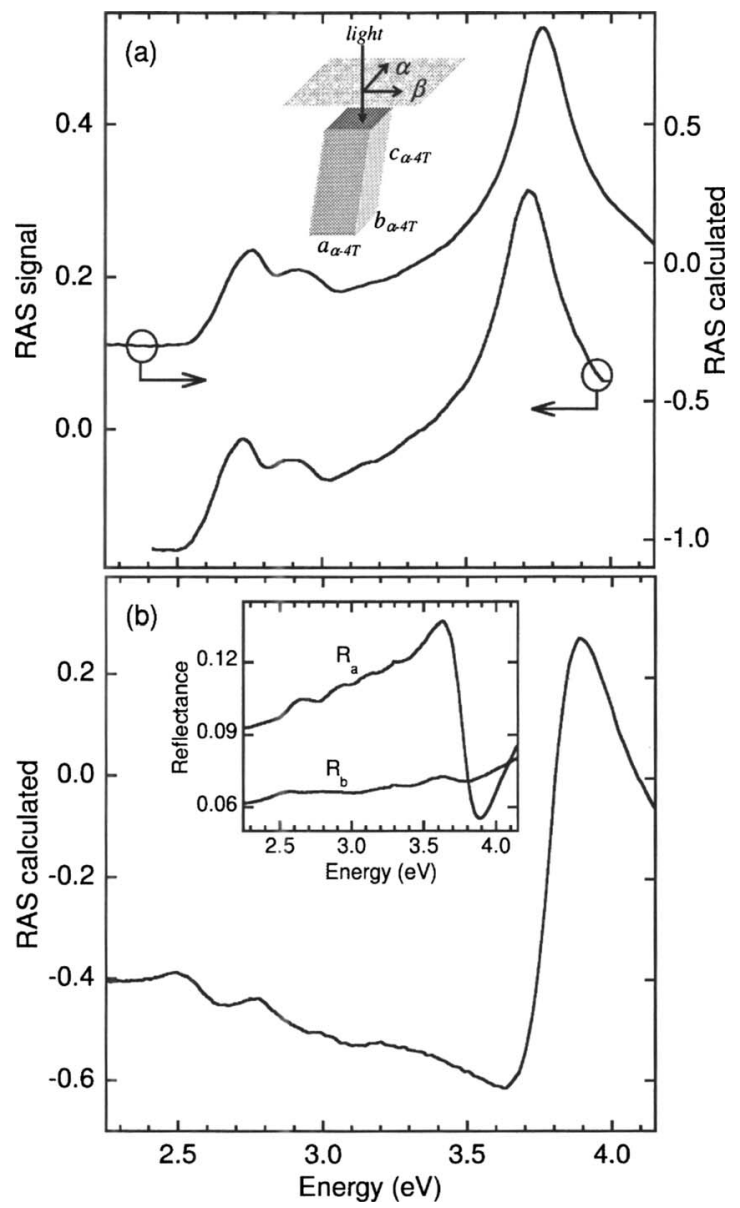

FIG. 1. (a) Experimental RAS spectrum of the $\alpha-4 \mathrm{~T} / \mathrm{LT}$ crystal (lower curve, left axis) and RAS spectrum calculated (upper curve, right axis) from reflectance spectra collected under polarized light on the same sample. In the inset, the sample orientation is sketched. (b) RAS spectrum of the $\alpha-4 \mathrm{~T} / \mathrm{LT}$ crystal calculated from reflectance spectra collected under polarized light (reported in the inset) for a sample lying on an index-matched glass plate.

a consequence, normal incidence optical measurements on the (001) plane permit one to fully detect the $b$-polarized transition, while-collecting $a$-polarized spectra-only the projection of the transition moment of the highest energy transition onto the (001) plane can be probed. ${ }^{20}$ Given this frame, in the spectral range from about 2 to $4 \mathrm{eV}$, characteristic spectral features related to $\alpha-4 \mathrm{~T}$ optical transitions can be detected in transmission and reflection spectra. ${ }^{21,22}$ Figure 1(a) shows the experimental RAS spectrum (lower curve) collected in situ on a $\alpha$-4T/LT single crystal placed on a silica substrate by modulating the electric field polarization along the $b$ axis and the $a$ axis of the crystal surface (see the sketch in the figure). For comparison, the spectrum calculated from experimental reflectance spectra $R_{b}$ and $R_{a}$ collected on the same sample with the electric field along the $b$ axis or the $a$ axis of the crystal (upper curve) is also reported. A very good agreement is evident, confirming that the proper optical response is detected without any artifacts when in situ measurements are carried out in the OMBE apparatus (slight discrepancies are present, but they are negligible at the level of our discussion due to the different experimental method, apparatus, and sample alignment). It should be noted, however, that RAS spectra in panel (a) do not represent the intrinsic response of the $\alpha-4 \mathrm{~T} / \mathrm{LT}$ single crystal. Indeed, the sharp edge at $2.6 \mathrm{eV}(\alpha-4 \mathrm{~T} / \mathrm{LT}$ absorption edge $)$ and the spectral line shape just beyond are the clear signature that we have collected light reflected at the crystal/glass substrate interface due to the finite thickness of the crystal, its low absorption coefficient in this spectral range, and the discontinuity between the refractive indices of the crystal and the glass substrate. In other words, while true reflectivity is a property of the air/crystal interface, reflectance (and RAS) spectra collected in this case probe the bulk properties of the $\alpha-4 \mathrm{~T} / \mathrm{LT}$ crystal together with the properties of the crystal/ glass interface. This induces marked differences when reflectivity and reflectance spectra are compared (for the $\alpha-4 \mathrm{~T} / \mathrm{LT}$ crystal, see Refs. 21 and 22).

To reproduce the intrinsic optical anisotropy of $\alpha-4 \mathrm{~T} / \mathrm{LT}$ single crystals, we have measured-with the same light polarization as before-reflectance spectra of the (001) surface of the $\alpha-4 \mathrm{~T}$ single crystal placed on a special high-index glass substrate. To avoid collection of light reflected at the crystal/substrate interface, the chosen substrate material was index matched with the $\alpha$-4T/LT crystal. In this way, the reflectance spectra $R_{b}$ and $R_{a}$ [reported in the inset of Fig. 1(b)] can be viewed as the true reflectivity of the crystal even though the crystal thickness is finite. In Fig. 1(b) we report the RAS spectrum calculated from these reflectance spectra. It represents the true anisotropy spectrum and mostly reflects the spectral features of the known $a$-polarized spectrum of $\alpha-4 \mathrm{~T} / \mathrm{LT}$ (Ref. 21) (for example, the negative peak at about $3.65 \mathrm{eV}$ ), while it does not reproduce completely the spectra of panel (a), where the mentioned interface-related features are present. As a matter of fact, for practical reasons it is not always possible to use index-matched substrates since for each organic crystal one should precisely know its refractive index and use the properly matched material: crystals are commonly placed on a silica glass substrate, in general, not index matched, and the effects of such a substrate should be taken into account.

In conclusion, RAS does not probe only the intrinsic optical properties of the anistropic crystal used as substrate but it also gives spectra fully reproducible from reflectance measurements, therefore reliable and useful as a reference of the "bare substrate" response in view of in situ studies of thin film growth.

\section{B. Homoepitaxy}

For a proper study of organic epitaxy by RAS, we have first investigated the OMBE growth of a material on a single crystal of the same material; in this situation, we expect that order and orientation of the overlayer are closely related to the crystal substrate. In Figs. 2 and 3, we present the results of AFM and RAS analysis of $\alpha-4 \mathrm{~T}$ film deposition by OMBE on $\alpha-4 \mathrm{~T}$ single crystals, demonstrating that these films grow homoepitaxially up to tens of monolayers. ${ }^{15}$

The homoepitaxial growth is evident through the analysis of AFM images taken ex situ on $\alpha-4 \mathrm{~T}$ films of different 

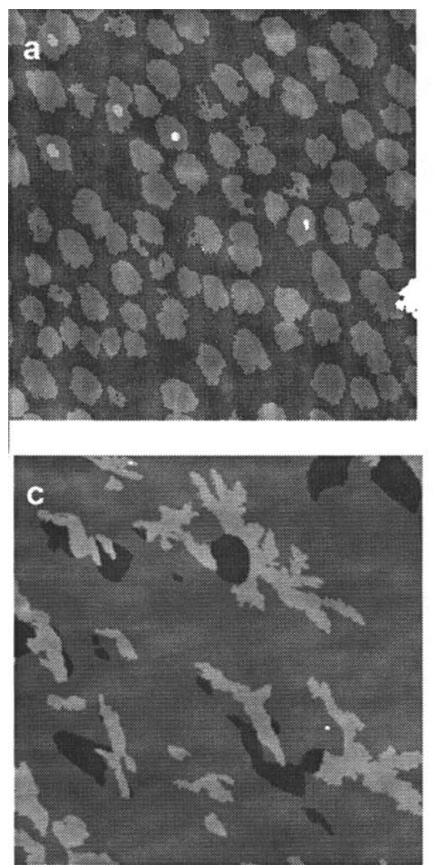
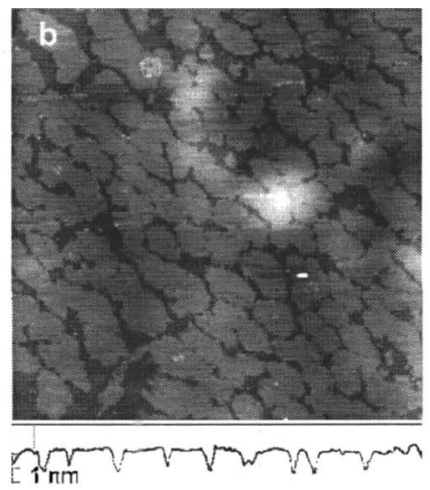

d
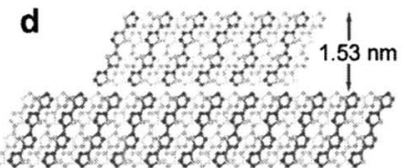

$4 \mathrm{~T}[100] \longrightarrow$

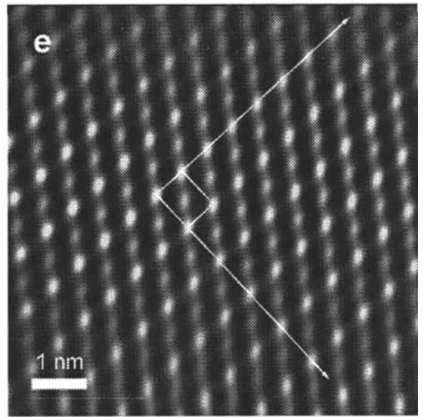

FIG. 2. (a)-(c) $20 \times 20 \mu \mathrm{m}^{2}$ AFM images collected on $\alpha$-4T films of different thicknesses $(1,2$, and 3 nm, respectively) grown on the $\alpha$-4T single crystal by OMBE; below panel (b), a cross-sectional profile of the AFM image taken along a horizontal line is reported. (d) Sketch of the molecular arrangement at the homoepitaxial interface as deduced by AFM images and RAS results. (e) Molecular resolution AFM image collected on the surface of the $\alpha$-4T film reported in (b), showing the film orientation and its surface unit cell.

thicknesses grown on the (001) face of $\alpha$-4T/LT single crystals. Selected AFM images are reported [Fig. 2(a)-2(c)] where monomolecular islands (1.5 $\mathrm{nm}$ thick) are observed on the substrate, whose coverage increases with film thickness according to a visible layer-by-layer growth. The profile of the AFM image in Fig. 2(b), reported below, shows the island thickness and permits sketching the structure of the film

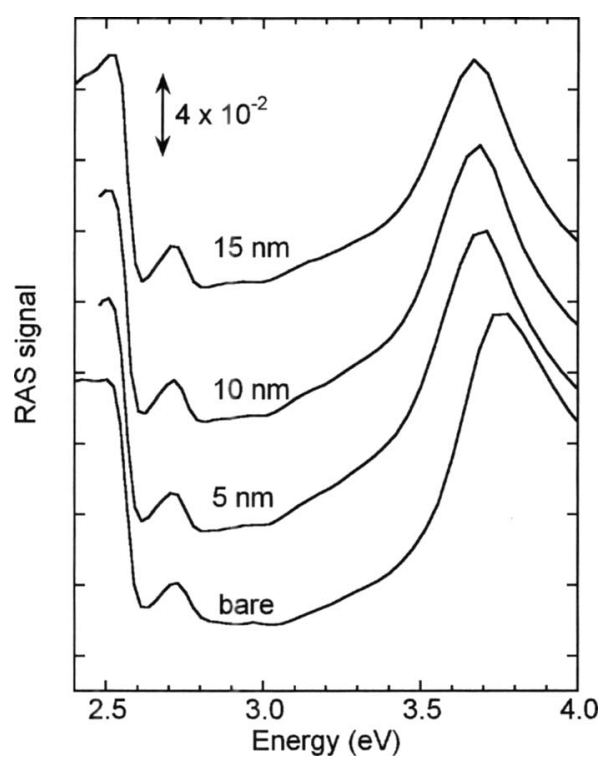

FIG. 3. RAS spectra collected in situ at different stages of the OMBE growth of the $\alpha$-4T film on the $\alpha$-4T single crystal. Each spectrum-labeled with the nominal film thickness, has been shifted along the vertical axis by +0.04 with respect to the previous one. growing on the single crystal [see Fig. 2(d)]. Finally, the AFM image reported in Fig. 2(e) has been collected with molecular resolution on an island of the $\alpha-4 \mathrm{~T}$ film in Fig. 2(b) and shows the surface unit cell and its local orientation; this demonstrates beyond any doubt the high crystallinity and unique orientation of the film.

However, AFM images (in air and at room temperature) suffer the limitation of being obtained after the complete sample growth and after the extraction from the OMBE chamber; this means that the images have been collected after a certain period of time from deposition under different environmental conditions. The application of RAS to monitor the evolution of the sample optical anisotropy during OMBE deposition allows to overcome this limitation. The RAS spectra in Fig. 3-measured during depositiondisplay a peculiar evolution of the line shape with film thickness from 0 (bare substrate) up to 5,10 , and finally $15 \mathrm{~nm}$. In the spectral region where $\alpha-4 \mathrm{~T}$ absorption is high (around $3.7 \mathrm{eV}$ and above), the spectra are nearly unchanged with increasing film thickness. The shift of the main peak evident by comparing the spectra of the bare substrate with the complete series could be originated from a different-although ordered-arrangement of the initially deposited $\alpha$-4T molecules with respect to the underlying substrate (see a more detailed discussion in Ref. 15). At about $2.6 \mathrm{eV}$, a sharp step is detected in all spectra, as already observed for a different sample in Fig. 1(a) (it is another sample with a different thickness), related to the absorption edge of $\alpha-4 \mathrm{~T} / \mathrm{LT}$. At lower photon energies, the amplitude of RAS signal exhibits a linear evolution with the film thickness [see also Fig. 1 of Ref. 15], related to the birefringence contribution from the 

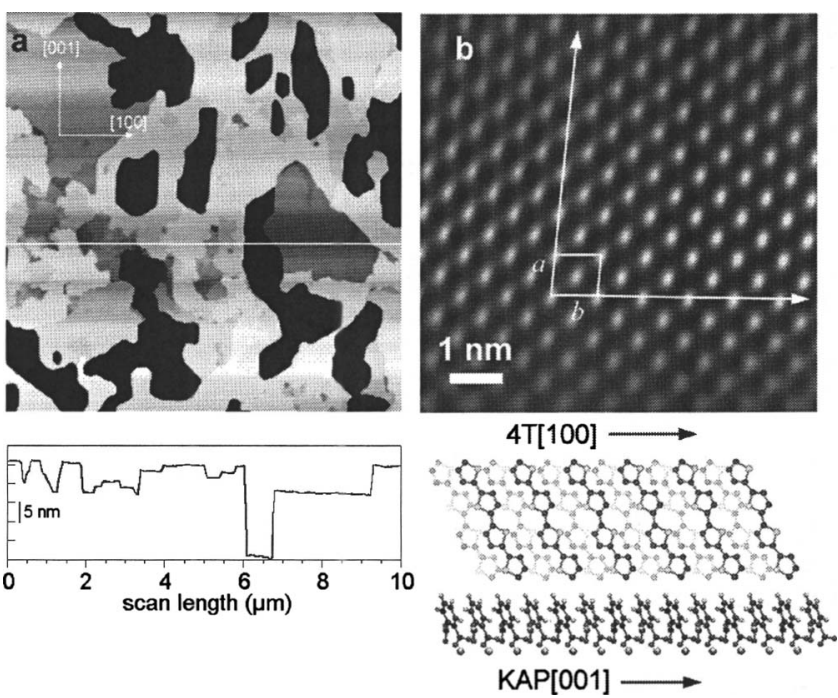

FIG. 4. (a) $10 \times 10 \mu \mathrm{m}^{2}$ AFM image collected on the $\alpha$-4T film (thickness of $10 \mathrm{~nm}$ ) grown on the $\mathrm{KAP}(010)$ single crystal by OMBE; the orientation of the substrate unit cell is indicated by white arrows; below the image, a cross-sectional profile taken along a horizontal line is reported. (b) Molecular resolution AFM image collected on the surface of the $\alpha$-4T film shown in (a), displaying the film orientation and the surface unit cell; below, the sketch of the molecular arrangement at the heteroepitaxial interface as deduced by AFM images is reported.

film, scaling indeed with thickness. The whole behavior observed, connected to the optical properties of the $\alpha$-4T crystal, is consistent with a perfect match between the film and the substrate crystal structure; the growth of an amorphous layer as well as of a crystalline film (but differently oriented) would produce a distinct evolution of the sample anisotropy with thickness. The spectra in Fig. 3 are thus the signature of a perfect homoepitaxial growth, in agreement with AFM, but detected during the film growth.

\section{Heteroepitaxy}

In organic heteroepitaxy, AFM demonstrates evidently the high quality of the interface formation and evolution [for details, see Refs. 2, 14, 23, and 24]. Figure 4(a) and the cross-sectional AFM profile reported below show the layered morphology of the $\alpha-4 \mathrm{~T}$ film grown by OMBE on $\mathrm{KAP}(010)$; the layer spacing value and the high resolution analysis performed on the film surface [Fig. 4(b)] reveal (001)-oriented domains, fulfilling the epitaxial relation in accordance with $\alpha-4 \mathrm{~T}[100] \| \mathrm{KAP}[001]{ }^{14}$ The sketch of the interface structure is shown below and illustrates the film alignment on KAP.

Similar to homoepitaxy, RAS has been applied to follow in real time the deposition process. In Fig. 5 RAS spectra collected at different stages of the $\alpha-4 \mathrm{~T}$ film growth on a KAP single crystal are shown. The substrate was kept at low temperature $(146 \mathrm{~K})$ during OMBE operation to obtain the thermodynamic conditions inducing the layer-by-layer growth. ${ }^{17,23}$ The three curves refer to the bare KAP substrate, a $4 \mathrm{~nm}$ thick film, and an $8 \mathrm{~nm}$ thick film. The KAP crystal in this spectral region is transparent, but, being anisotropic, ${ }^{25}$

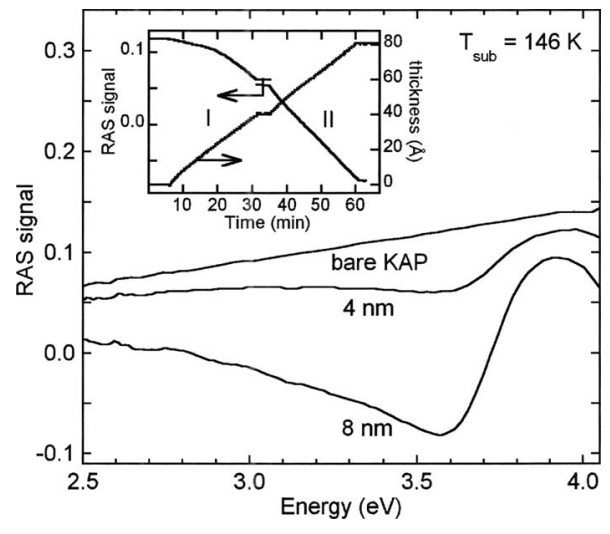

FIG. 5. RAS spectra collected in situ at different stages of the growth of the $\alpha-4 \mathrm{~T}$ film on the KAP single crystal at $146 \mathrm{~K}$. The spectra have been labeled with the corresponding nominal film thickness. Inset: time evolution of the RAS signal at $3.56 \mathrm{eV}$ (left axis) and of the film thickness as measured by the calibrated quartz microbalance (right axis) during the growth steps I and II (see text).

it gives a non-negligible RAS response increasing monotonically up to the absorption edge slightly, above $4 \mathrm{eV}$. After deposition of $4 \mathrm{~nm}$ of $\alpha-4 \mathrm{~T}$, a negative peak arises at about $3.55 \mathrm{eV}$, whose amplitude increases with thickness. This is interpreted as the signature of an oriented thin film that exposes the (001) face as contact plane with the unit cell axis $a_{\alpha-4 \mathrm{~T}}\left(b_{\alpha-4 \mathrm{~T}}\right)$ aligned to $c_{\mathrm{KAP}}\left(a_{\mathrm{KAP}}\right)$ axis [in this experiment the $\alpha$ direction of the RAS apparatus is nearly parallel to $a_{\mathrm{KAP}}$ and the RAS signal is defined as in Eq. (1)]. By comparison with the optical properties of crystalline $\alpha-4 \mathrm{~T}$ [see also Fig. 1(b)], this structure is shifted by about $0.1 \mathrm{eV}$ toward lower photon energies; it can therefore be attributed to the growth of a different pseudomorphic $\alpha$-4T phase, favored by a peculiar epitaxial relation to the cooled KAP. Indeed, a good match with substrate is reached by a slight tilt of the molecules in the crystalline film with respect to the equilibrium LT structure of $\alpha$-4T. In particular, such a modified pseudomorphic structure leads to a stronger coupling of light with the main optical excitonic transition of $\alpha-4 \mathrm{~T}$, shifting the main peak, usually observed at about $3.7 \mathrm{eV}$.

In the inset of Fig. 5, we report the time evolution of the RAS signal measured at $3.55 \mathrm{eV}$ (maximum of the $\alpha-4 \mathrm{~T}$ related peak) during the two steps of deposition (steps I and II); during each step (about $23 \mathrm{~min}$ ), a nominal $4 \mathrm{~nm}$ thickness was deposited, monitored by the calibrated quartz balance (whose signal has been reported in the inset of Fig. 5 as well). The $\alpha-4$ T beam density was stable during the whole deposition, with all conditions being kept constant. During step I, the signal decreases with a continuously varying slope; this indicates a change in the efficiency of the molecular beam in growing the crystalline film. Even if the amount of molecules reaching the substrate per unit time (beam density) is constant, the growth rate of the crystalline layer varies. On the contrary, during step II, the signal varies linearly, indicating a constant growth rate, which is the same as the final one of step I. On this basis, we identify two different growth regimes: (i) the deposition of the first nominal $3 \mathrm{~nm}$, that is, the condensation of molecules on the bare KAP sub- 
strate and the simultaneous development of $\alpha$-4T crystalline islands; and (ii) after the first layer has been completed, the $\alpha$-4T film grows on the $\alpha$-4T crystalline layer with the constant growth rate characteristic of $\alpha$-4T homoepitaxy.

\section{CONCLUSIONS}

In this article we have presented and discussed-through paradigmatic and significant examples - a strategy to efficiently monitor the deposition in UHV by using RAS in situ. Given the anisotropic optical properties of most organic materials, the choice of RAS could be considered the most proper in spite of some limitations to a quantitative interpretation. We have demonstrated, by a careful phenomenological analysis of different samples, by a smart choice of the substrates and of the thermodynamic conditions of deposition and by comparison with AFM results, that the epitaxial growth of organic layers can be successfully monitored and studied, opening relevant perspectives for the future synergy between OMBE and RAS.

\section{ACKNOWLEDGMENTS}

One of the authors (G.B.) kindly acknowledges CHOSE for partial financial support.

${ }^{1}$ Physics of Organic Semiconductors, edited by W. Brütting (Wiley-VCH, Weinheim, 2005).

${ }^{2}$ A. Sassella, M. Campione, and A. Borghesi, Riv. Nuovo Cimento 31, 457 (2008).

${ }^{3}$ S. R. Forrest, Chem. Rev. (Washington, D.C.) 97, 1793 (1997).

${ }^{4}$ P. Weightmann, D. S. Martin, R. J. Cole, and T. Farrel, Rep. Prog. Phys. 68, 1251 (2005).

${ }^{5}$ S. Selci, F. Ciccacci, G. Chiarotti, P. Chiaradia, and A. Cricenti, J. Vac. Sci. Technol. A 5, 327 (1987); P. Chiaradia and G. Chiarotti, in Photonic
Probes of Surfaces, edited by P. Halevi (Elsevier Science, New York, 1995).

${ }^{6}$ C. Goletti, G. Bussetti, P. Chiaradia, A. Sassella, and A. Borghesi, Appl. Phys. Lett. 83, 4146 (2003).

${ }^{7}$ L. D. Sun, M. Hohage, P. Zeppenfeld, S. Berkebile, G. Koller, F. P. Netzer, and M. G. Ramsey, Appl. Phys. Lett. 88, 121913 (2006).

${ }^{8}$ H. Proehl, T. Dienel, R. Nitsche, and T. Fritz, Phys. Rev. Lett. 93, 097403 (2004); O. Pluchery, R. Coustel, N. Witkowski, and Y. Borensztein, J. Phys. Chem. B 110, 22635 (2006).

${ }^{9}$ R. Forker and T. Fritz, Phys. Chem. Chem. Phys. 11, 2142 (2009).

${ }^{10}$ S. Trabattoni, S. Laera, R. Mena, A. Papagni, and A. Sassella, J. Mater. Chem. 14, 171 (2004).

${ }^{11}$ M. Campione, R. Ruggerone, S. Tavazzi, and M. Moret, J. Mater. Chem. 15, 2437 (2005).

${ }^{12}$ T. Siegrist, C. Kloc, R. A. Laudise, H. E. Katz, and R. C. Haddon, Adv. Mater. (Weinheim, Ger.) 10, 379 (1998).

${ }^{13}$ M. Campione, M. Cartotti, E. Pinotti, A. Sassella, and A. Borghesi, J. Vac. Sci. Technol. A 22, 482 (2004).

${ }^{14}$ M. Campione et al., J. Am. Chem. Soc. 128, 13378 (2006).

${ }^{15}$ A. Sassella, A. Borghesi, M. Campione, S. Tavazzi, C. Goletti, G. Bussetti, and P. Chiaradia, Appl. Phys. Lett. 89, 261905 (2006).

${ }^{16}$ J. D. E. McIntyre and D. E. Aspnes, Surf. Sci. 24, 417 (1971).

${ }^{17}$ A. Sassella, M. Campione, M. Moret, A. Borghesi, C. Goletti, G. Bussetti, and P. Chiaradia, Phys. Rev. B 71, 201311(R) (2005).

${ }^{18}$ M. Campione, A. Borghesi, M. Laicini, A. Sassella, G. Bussetti, C. Goletti, and P. Chiaradia, J. Chem. Phys. 127, 244703 (2007).

${ }^{19}$ A. S. Davydov, Theory of Molecular Excitons (Plenum, New York, 1971).

${ }^{20}$ M. Laicini, P. Spearman, S. Tavazzi, and A. Borghesi, Phys. Rev. B 71, 045212 (2005).

${ }^{21}$ M. Laicini, P. Spearman, L. Raimondo, S. Trabattoni, L. Miozzo, and M. Moret, J. Lumin. 110, 207 (2004).

${ }^{22}$ S. Tavazzi, A. Borghesi, M. Campione, M. Laicini, P. Spearman, and S. Trabattoni, J. Chem. Phys. 120, 7136 (2004).

${ }^{23}$ A. Sassella, M. Campione, A. Papagni, C. Goletti, G. Bussetti, P. Chiaradia, V. Marcon, and G. Raos, Chem. Phys. 325, 193 (2006).

${ }^{24}$ M. Campione, L. Raimondo, and A. Sassella, J. Phys. Chem. C 111, 19009 (2007)

${ }^{25}$ A. Sassella, A. Borghesi, Th. Wagner, and J. Hilfiker, J. Appl. Phys. 90, 3838 (2001). 